\title{
Posterior Interosseous Nerve of the Elbow
}

\section{Normal Appearances Simulating Entrapment}

\author{
Qian Dong, MD, David A. Jamadar, MB, BS, \\ Brian L. Robertson, AAS, RDMS, Jon A. Jacobson, MD, \\ Elaine M. Caoili, MD, Thomas Gest, PhD, Gandikota Girish, MB, BS
}

\begin{abstract}
Abbreviations
ANOVA, analysis of variance; AP, anteroposterior; PIN, posterior interosseous nerve
\end{abstract}

Received November 11, 2009, from the Department of Radiology, University of Michigan Hospital, Ann Arbor, Michigan USA (Q.D., D.A.J., B.L.R., J.A.J., E.M.C., G.G.); and Division of Anatomical Sciences, University of Michigan Medical School, Ann Arbor, Michigan USA (T.G.). Revision requested December 18, 2009. Revised manuscript accepted for publication December 30, 2009.

Address correspondence to Qian Dong, MD, Department of Radiology, University of Michigan Hospital, 1500 E Medical Center Dr, Ann Arbor, MI 48109 USA.

E-mail: bingch@med.umich.edu

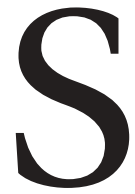

onography has proven useful in the diagnosis of a variety of abnormalities affecting peripheral nerves. ${ }^{1,2}$ Evaluation for posttraumatic neuroma after amputation is a well-known application for sonography. ${ }^{3}$ Other applications include ulnar nerve evaluation at the elbow for enlargement and subluxation, the median nerve for carpal tunnel syndrome, and the tibial nerve to access for entrapment at the ankle. ${ }^{2-5}$ The ability of high-frequency transducers to display neurologic structures in exquisite detail coupled with a dynamic component to the study makes sonography an attractive adjunct to clinical evaluation. ${ }^{1,6}$

Among the upper extremity entrapment neuropathies, posterior interosseous nerve (PIN) syndrome (or supinator syndrome) has been described using magnetic resonance imaging and sonography. ${ }^{7}$ In this condition, the PIN may be entrapped as it courses under the arcade of Frohse and enters the supinator muscle (Figure 1). The hallmark of such nerve entrapment is abnormal swelling 
of the involved nerve and an abrupt caliber change at the entrapment site.

In our clinical practice, we have observed on sonography an abrupt caliber change of the PIN in the long axis at the arcade of Frohse as it passes into the supinator muscle in asymptomatic individuals. We believe that this could be a potential pitfall prompting misdiagnosis of an entrapment syndrome. The purpose of this study was to characterize the normal sonographic appearance of the PIN in asymptomatic individuals.

\section{Materials and Methods}

Institutional Review Board approval was obtained before commencing this retrospective study with informed consent waived. Sixty-four patients with sonographic examinations of the elbows from January 2007 to December 2008 were evaluated. Patients were referred for sonography of the elbow to evaluate soft tissue masses,

Figure 1. Anterior elbow showing the course of the radial nerve. Posterior interosseous nerve entrapment may occur because of a prominent radial recurrent artery (RRA), medial edge of extensor carpi radialis brevis (ECRB), and proximal edge of the supinator muscle (SP; arcade of Frohse). RN indicates radial nerve; and SRN, superficial branch of radial nerve.

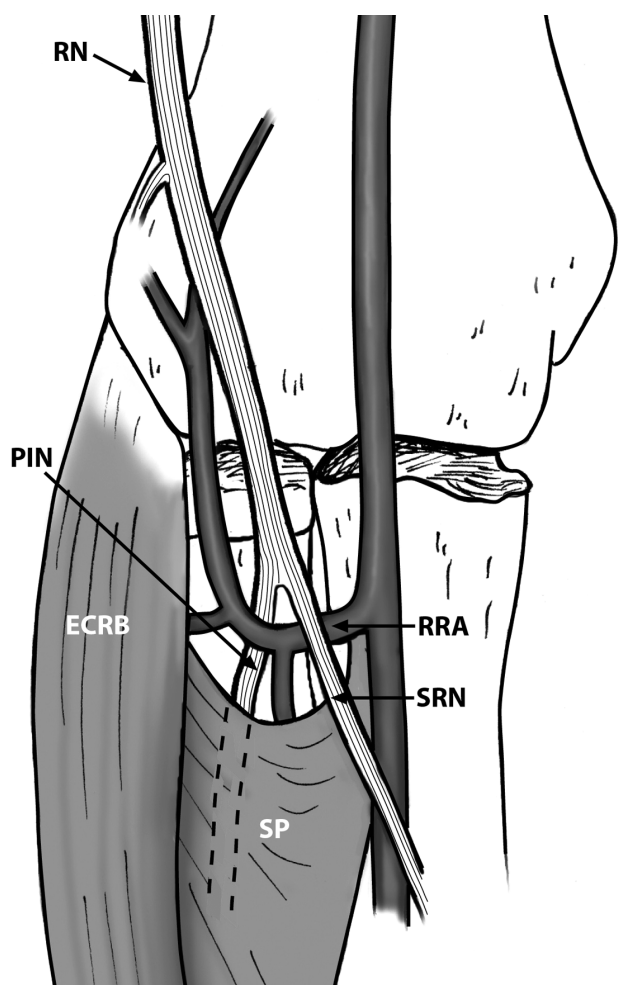

to evaluate ulnar nerve symptoms and document snapping ulnar nerve syndrome, and to identify medial and lateral epicondylitis, distal biceps tendon injury, olecranon bursitis, and synovitis. Seventeen patients were excluded from the study because there was a history of severe elbow trauma or surgery, median or radial nerve neuropathy, or anterior lateral elbow pain or the PIN was not adequately imaged. This resulted in 50 elbows of 47 patients; in 3 patients, both elbows were asymptomatic, noted at the time of the sonographic examination.

Sonographic examinations were performed using a 5- to 17-MHz compact linear array transducer (iU22; Philips Healthcare, Bothell, WA) by a diagnostic medical sonographer (10 years of experience in performing only musculoskeletal sonographic examinations), supervised by 1 of 10 fellowship-trained musculoskeletal radiologists (range of experience from 2-11 years), as part of routine patient care. Sonography consisted of both static gray scale images and cine clips stored in the picture archiving computer system.

Sonograms were retrospectively reviewed by the sonographer who performed the sonographic examinations, supervised by 1 of the fellowshiptrained musculoskeletal radiologists. The PIN (or deep branch of the radial nerve) was identified in a cross section as an oval structure deep to the brachioradialis, relatively hypoechoic when compared with adjacent muscle, surrounded by a thin relatively hyperechoic layer of connective tissue. In the long axis, the nerve appeared as a hypoechoic tubular structure characteristically coursing distally into the supinator muscle (Figure 2).

Figure 2. Sonogram from a 34-year-old asymptomatic man showing the hypoechoic PIN in the long axis (arrowheads) with tapering as it passes into the supinator muscle (stars) and under the arcade of Frohse (open arrowhead). Arrows indicate the cortex of the proximal radius.

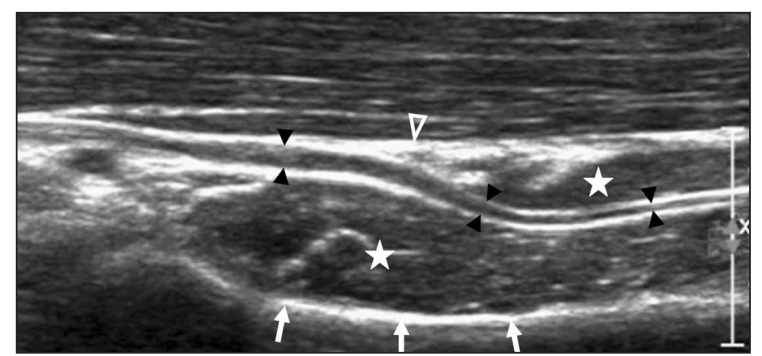


The short-axis cross-sectional area, using the circumferential trace technique, and anteroposterior (AP) dimension of the PIN were evaluated immediately proximal to the arcade of Frohse, identified as a linear hyperechoic area, just before the nerve entered the supinator muscle and $1 \mathrm{~cm}$ proximal and $1 \mathrm{~cm}$ distal to this point (Figure 3). Statistical analysis included an analysis of variance (ANOVA) test to determine significant differences between the cross-sectional areas at the 3 measured points.

In addition to cross-sectional area and AP measurements of the PIN, branching of the PIN if present was recorded. To further study the anatomy of the radial nerve at the elbow, anatomic dissection of an unembalmed cadaveric elbow was also completed by one of the authors.

\section{Results}

The 50 elbows (31 right and 19 left) were from 47 patients (24 men and 23 women) of ages ranging from 19 to 74 years (mean, 47.3 years; SD, 16.38 years). The cadaveric elbow was from an 89 -yearold woman.

The mean-cross sectional area of the PIN immediately proximal to the arcade of Frohse was $0.019 \mathrm{~cm}^{2}$ (SD, $\left.0.00729 \mathrm{~cm}^{2}\right) ; 1 \mathrm{~cm}$ proximal to the arcade, it was $0.016 \mathrm{~cm}^{2}$ (SD, $0.00387 \mathrm{~cm}^{2}$ ); and $1 \mathrm{~cm}$ distal to the arcade, it was $0.018 \mathrm{~cm}^{2}$ (SD, $0.02110 \mathrm{~cm}^{2}$ ). A repeated measures ANOVA test showed no significant differences between the cross-sectional areas of the PIN at all 3 levels $(P=.59)$.

The mean AP thickness of the PIN immediately proximal to the arcade of Frohse was $0.108 \mathrm{~cm}$ (SD, $0.03178 \mathrm{~cm}$ ); $1 \mathrm{~cm}$ proximal to the arcade, it was $0.123 \mathrm{~cm}$ (SD, $0.02172 \mathrm{~cm}$ ); and $1 \mathrm{~cm}$ distal to the arcade, it was $0.069 \mathrm{~cm}(\mathrm{SD}, 0.02074 \mathrm{~cm})$. A repeated measures ANOVA test showed a significant difference between the AP thickness of the PIN distal to the arcade when compared with the values at the arcade and proximal to the arcade $(P<.0001$; Figure 4$)$.

Two of the 50 elbows showed bifurcation of the PIN within the supinator muscle (Figure 5). Dissection of the cadaveric elbow showed the radial nerve bifurcating to the superficial and deep branches. The deep branch of the radial nerve, or PIN, was then identified coursing beneath the arcade of Frohse and between the 2 layers of the supinator muscle (Figure 6).

\section{Discussion}

A caliber change of an enlarged peripheral nerve can be an imaging sign of nerve entrapment. In our study, the normal PIN showed a caliber change as it entered into the supinator muscle; however, this caliber change was in the AP dimension without a true change in the nerve area as seen in the short axis. This normal finding could potentially be misinterpreted as nerve compression when imaging in the long axis.

The radial nerve is the largest branch of the brachial plexus and arises from its posterior cord. It passes obliquely across the posterior aspect of the humerus and pierces the lateral intermuscular septum to enter the anterior compartment of the arm above the elbow. The radial nerve then passes between the brachialis and brachioradialis muscles, continuing anterior to the lateral epicondyle, where it divides into the deep branch (or PIN) and superficial branch. The PIN descends deep in relation to the proximal edge of the superficial layer of the supinator muscle, which is known as the arcade of Frohse (Figure 6) and courses distally, providing innervation to the extrinsic wrist extensor muscles except for the extensor carpi radialis longus. ${ }^{8}$

The PIN may undergo compression or entrapment at a number of sites, with the arcade of Frohse being the most common site. ${ }^{8-10}$ Other structures can potentially cause compression or entrapment, including the medial edges of the extensor carpi radialis brevis, fibrous bands at the radial head, and the leash of Henry where the lateral branches of the recurrent radial artery cross the nerve (Figure 1). ${ }^{9,10}$

A caliber change of the PIN as it passes through the arcade of Frohse may be considered a manifestation of nerve compression or entrapment. ${ }^{7,11,12}$ In our study, the observation was made that the PIN became flattened as it pierced the proximal supinator muscle in the forearm (Figure 4). In all elbows, the PIN showed a decrease in the AP dimension once within the proximal supinator muscle at the level of the arcade of Frohse. When imaging in the short axis 
Figure 3. Images from a 47-year-old asymptomatic woman. A, Sonogram of the PIN in the long axis showing measurement locations just proximal to the arcade of Frohse (middle circle), $1 \mathrm{~cm}$ proximal (left circle), and $1 \mathrm{~cm}$ distal (right circle). B-D, Sonograms of the PIN in cross section showing area measurements from proximal to distal. E-G, Sonograms of the PIN in cross section showing AP measurements from proximal to distal.

\section{B}

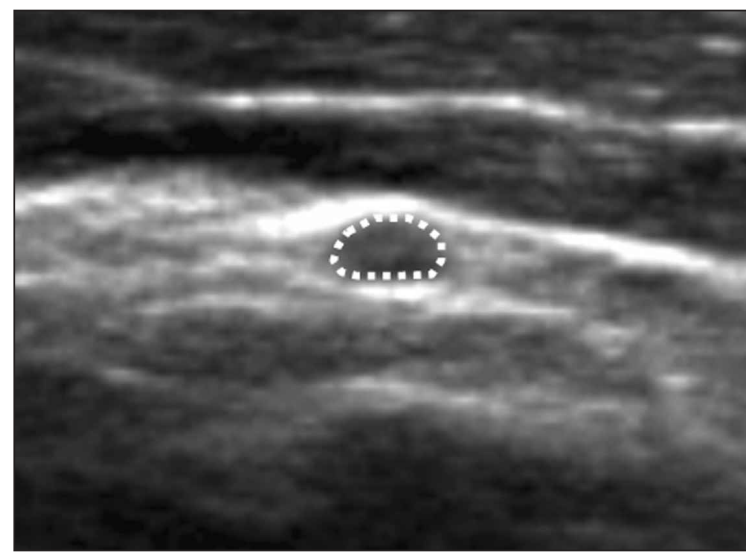

D

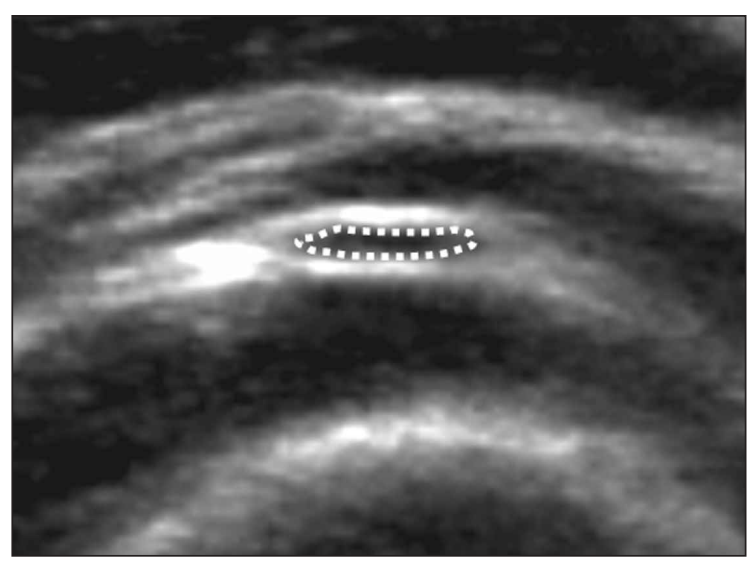

$\mathbf{F}$

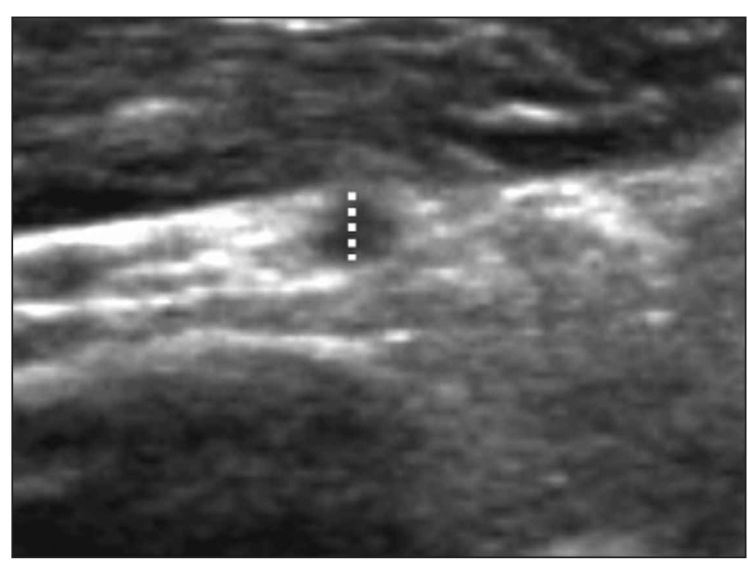

A

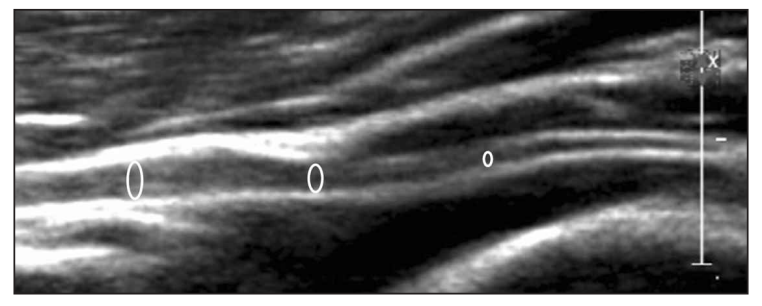

C

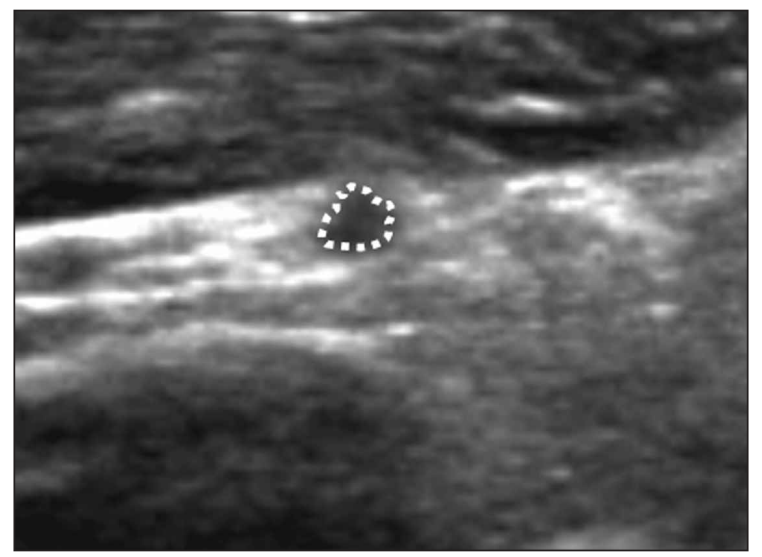

E

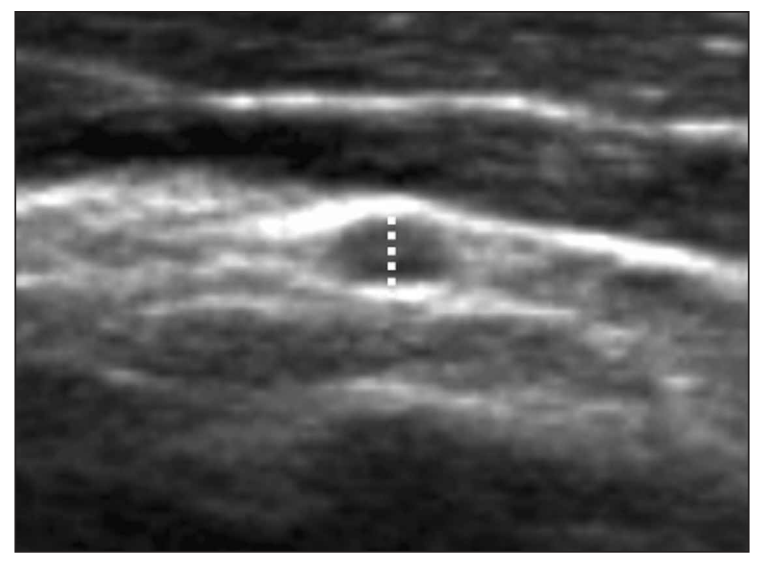

$\mathbf{G}$

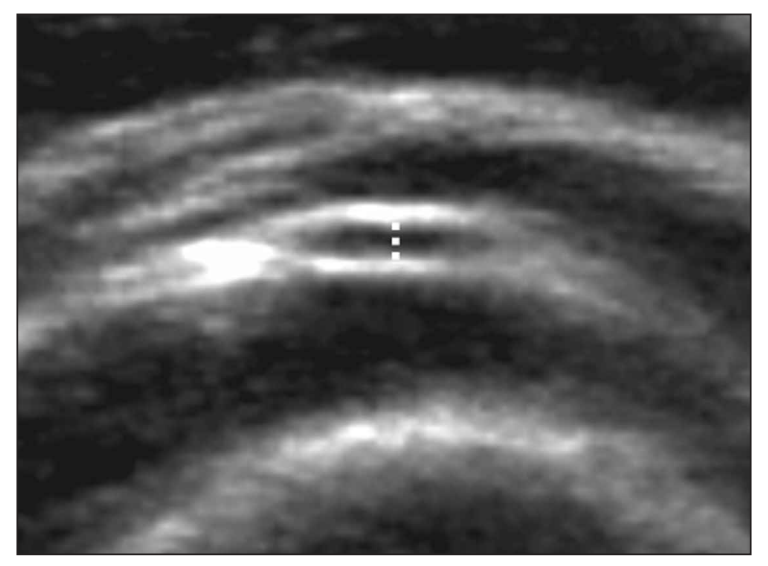


with assessment of the cross-sectional area, however, the PIN showed variability in volume along its course without statistical significance. As a result, the sonographic appearance of abrupt caliber reduction of the PIN at the arcade of Frohse may be a potential pitfall in assessing for PIN compression or entrapment when imaging in the long axis of the nerve. Cross-sectional measurements of the PIN in the axial plane provide more accurate information in evaluating

Figure 4. Images from a 46-year-old asymptomatic man. A, Sonogram showing the PIN in the long axis (arrowheads) as it passes under the arcade of Frohse and into the supinator muscle (stars). Arrows indicate the cortex of the proximal radius. $\mathbf{B}$ and $\mathbf{C}$, Sonograms of the PIN in the short axis (arrows) proximal (B) and distal (C) to the arcade of Frohse showing the caliber change in the AP dimension.

\section{A}

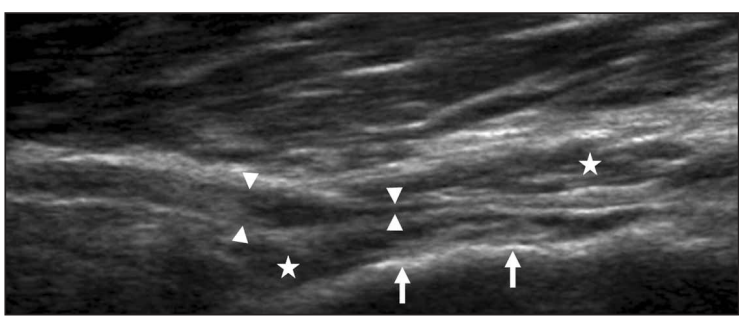

B

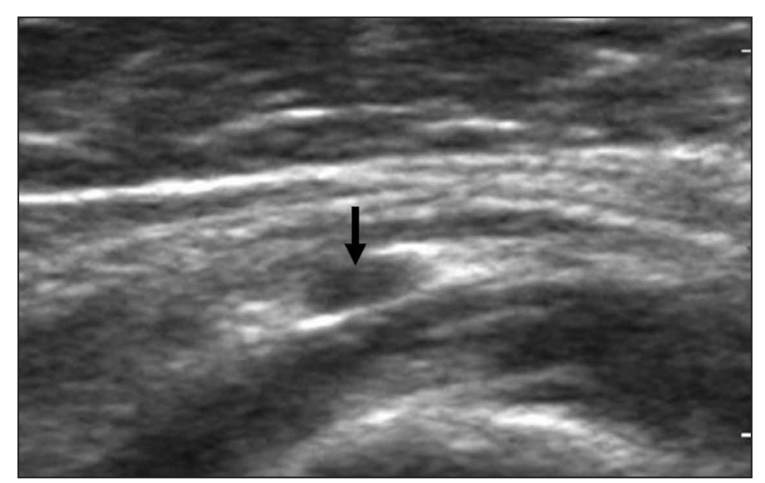

c

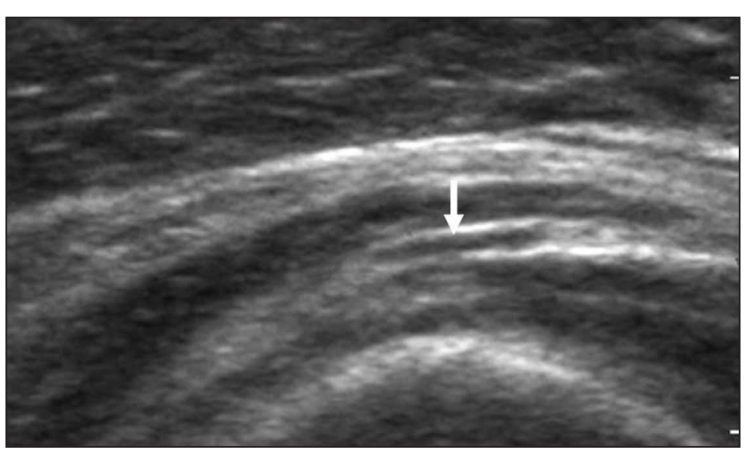

true caliber changes sonographically. Two of the 50 nerves evaluated showed bifurcation within the supinator muscle (Figure 5).

One of our patients presented with radial nerve symptoms and was excluded from the study. However, this patient was found to have entrapment of the PIN at the arcade of Frohse (Figure 7). In the long axis, the nerve was initially of normal caliber; then it became focally thickened just proximal to the point of entrapment; and then it was of normal caliber after the entrapment. This change in dimension is more dramatic than the normal variation described in this study and is emphasized by the measurements of the abnormal nerve when compared with the normal measurements in our study (cross-sectional area of the abnormal nerve immediately proximal to

Figure 5. Sonogram in the short axis from a 50-year-old asymptomatic man showing bifurcation of the PIN (arrows) within the supinator muscle. $R$ indicates proximal radius.

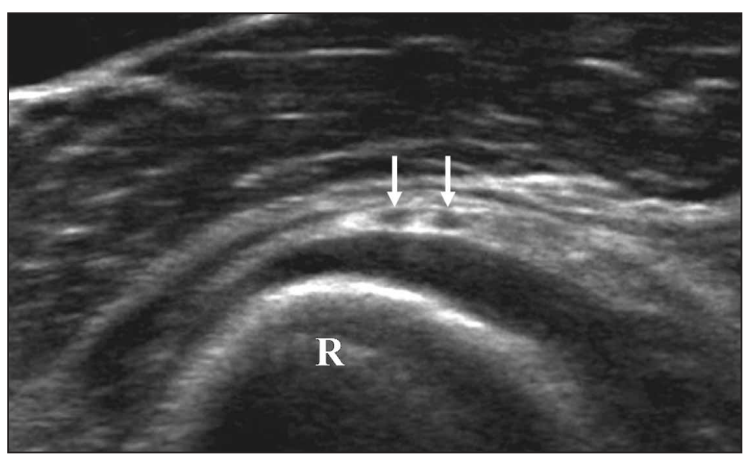

Figure 6. Dissection of an 89-year-old female cadaveric elbow showing the PIN (arrows) passing under the proximal edge (arrowheads) of the superficial layer of the supinator muscle (star), which is known as the arcade of Frohse. The superficial branch of the radial nerve (open arrows) is also shown.

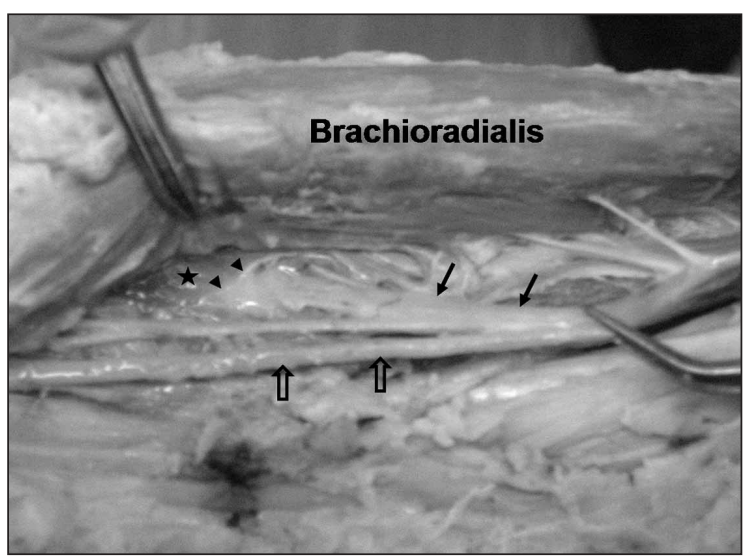




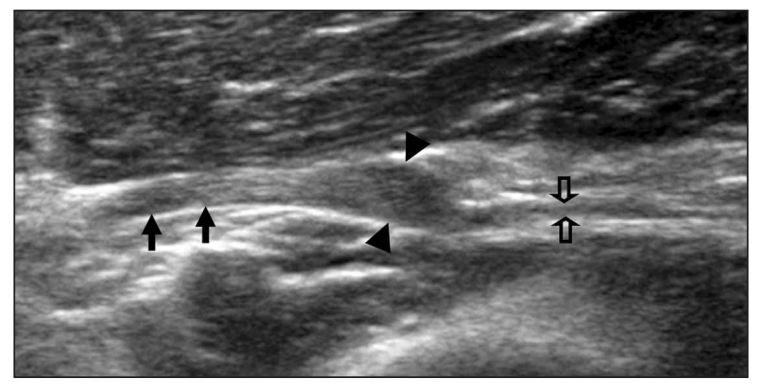

Figure 7. Sonogram from a 71-year-old woman with radial nerve symptoms showing the radial nerve and PIN in the long axis. The nerve was initially of normal caliber (arrows); then it became focally thickened (arrowheads) just proximal to the point of entrapment; and then it was of normal caliber after the entrapment (open arrows).

the arcade of Frohse, $0.092 \mathrm{~cm}^{2} ; 1 \mathrm{~cm}$ proximal to the arcade, $0.013 \mathrm{~cm}^{2}$; and $1 \mathrm{~cm}$ distal, 0.021 $\mathrm{cm}^{2}$ ). The abnormal nerve is focally enlarged just proximal to the compression when compared with its cross-sectional area proximal and distal, whereas the normal nerve appears to change caliber as it enters the supinator.

We acknowledge several limitations of the study. The retrospective nature of the study was one limitation, as well as the small size of the PIN, which both could introduce measurement error; however, all measurements were made by 1 individual (B.L.R.), and cine loops were used to find the optimal measurement locations. In addition, we relied on the patient history to determine whether the elbow was asymptomatic.

In summary, normal flattening of the PIN as it enters into the supinator muscle should not be misinterpreted as nerve entrapment as a caliber change is only in the AP dimension without a change in the nerve area.

\section{References}

1. Chiou HJ, Chou YH, Chiou SY, Liu JB, Chang CY. Peripheral nerve lesions: role of high-resolution US. Radiographics 2003; 23:e15. http://radiographics.rsna.org/content/23/6/ e15.long.

2. Martinoli C, Bianchi S, Gandolfo N, Valle M, Simonetti S, Derchi LE. US of nerve entrapments in osteofibrous tunnels of the upper and lower limbs. Radiographics 2000; 29(special issue):S199-\$213.

3. Ernberg LA, Adler RS, Lane J. Ultrasound in the detection and treatment of a painful stump neuroma. Skeletal Radiol 2003; 32:306-309.
4. Okamoto M, Abe M, Shirai H, Ueda N. Morphology and dynamics of the ulnar nerve in the cubital tunnel: observation by ultrasonography. J Hand Surg Br 2000; 25:85-89.

5. Wong SM, Griffith JF, Hui AC, Lo SK, Fu M, Wong KS Carpal tunnel syndrome: diagnostic usefulness of sonography. Radiology 2004; 232:93-99.

6. Peer S, Bodner G, Meirer R, Willeit J, Piza-Katzer H. Examination of postoperative peripheral nerve lesions with high-resolution sonography. AJR Am J Roentgenol 2001; 177:415-419.

7. Chien AJ, Jamadar DA, Jacobson JA, Hayes CW, Louis DS. Sonography and MR imaging of posterior interosseous nerve syndrome with surgical correlation. AJR Am J Roentgenol 2003; 181:219-221.

8. Barnum M, Mastey RD, Weiss AC, Akelman E. Radial tunnel syndrome. Hand Clin 1996; 12:679-689.

9. Konjengbam M, Elangbam J. Radial nerve in the radial tunnel: anatomic sites of entrapment neuropathy. Clin Anat 2004; 17:21-25.

10. Ferdinand $B D$, Rosenberg $Z S$, Schweitzer ME, et al. MR imaging features of radial tunnel syndrome: initial experience. Radiology 2006; 240:161-168.

11. Hill S, Hall S. Microscopic anatomy of the posterior interosseous and median nerves at the sites of potential entrapment in the forearm. J Hand Surg Br 1999; 24:170176.

12. Carr D, Davis P. Distal posterior interosseous nerve syndrome. J Hand Surg Am 1985; 10:873-878. 